\title{
Measuring ${ }^{13} \mathrm{C} /{ }^{15} \mathrm{~N}$ chemical shift anisotropy in $\left[{ }^{13} \mathrm{C},{ }^{15} \mathrm{~N}\right]$ uniformly enriched proteins using CSA amplification
}

\author{
Ivan Hung ${ }^{\mathrm{a}}$, Yuwei Ge ${ }^{\mathrm{a}, \mathrm{b}}$, Xiaoli Liu ${ }^{\mathrm{b}}$, Mali Liu ${ }^{\mathrm{b}}$, Conggang Li ${ }^{\mathrm{b} *}$, Zhehong Gan ${ }^{\mathrm{a} *}$ \\ ${ }^{a}$ Center of Interdisciplinary Magnetic Resonance, National High Magnetic Field Laboratory, 1800 East \\ Paul Dirac Drive, Tallahassee, FL 32310, USA \\ ${ }^{\mathrm{b}}$ Key Laboratory of Magnetic Resonance in Biological Systems, State Key Laboratory of Magnetic \\ Resonance and Atomic and Molecular Physics, National Center for Magnetic Resonance in Wuhan, Wuhan \\ Institute of Physics and Mathematics, Chinese Academy of Sciences, Wuhan, 430071 (P.R. China). \\ *Corresponding authors: gan@magnet.fsu.edu (Z. Gan), conggangli@wipm.ac.cn (C. Li)
}

\begin{abstract}
Extended chemical shift anisotropy amplification (xCSA) is applied for measuring ${ }^{13} \mathrm{C} /{ }^{15} \mathrm{~N}$ chemical shift anisotropy (CSA) of uniformly labeled proteins under magic-angle spinning (MAS). The amplification sequence consists of a sequence of $\pi$-pulses that repetitively interrupt MAS averaging of the CSA interaction. The timing of the pulses is designed to generate amplified spinning sideband manifolds which can be fitted to extract CSA parameters. The ${ }^{13} \mathrm{C} /{ }^{13} \mathrm{C}$ homonuclear dipolar interactions are not affected by the $\pi$ pulses due to the bilinear nature of the spin operators and are averaged by MAS in the xCSA experiment. These features make the constant evolution-time experiment suitable for measuring CSA of uniformly labeled samples. The incorporation of xCSA with multidimensional ${ }^{13} \mathrm{C} /{ }^{15} \mathrm{~N}$ correlation is demonstrated with a GB1 protein sample as a model system for measuring ${ }^{13} \mathrm{C} /{ }^{15} \mathrm{~N}$ CSA of all backbone ${ }^{15} \mathrm{NH},{ }^{13} \mathrm{CA}$ and ${ }^{13} \mathrm{CO}$ sites.
\end{abstract}

Keywords: chemical shift anisotropy, CSA, magic-angle spinning, MAS, spinning sidebands, magic-angle turning, MAT, CSA amplification, extended chemical shift modulation, protein, GB1

\section{Introduction}

Magic-angle spinning (MAS) is an ubiquitous method for obtaining highresolution nuclear magnetic resonance (NMR) spectra of solids. Rapid sample spinning averages line broadening arising from anisotropic spin interactions, such as chemical shift anisotropy (CSA) and dipolar coupling, yielding high-resolution spectra of powder samples. These anisotropic spin interactions are of great interest because of their direct 
relation to molecular structure and local electronic environment. In the presence of molecular motion, measurement of the scaled chemical shift anisotropy and dipolar coupling can also yield order parameters from the molecular dynamics. Numerous NMR techniques have been developed to reintroduce/recouple CSA or dipolar coupling under fast MAS, which is particularly useful for uniformly labeled proteins in conjunction with multi-dimensional correlation experiments because it can be used under high resolution conditions. However, the presence of ${ }^{13} \mathrm{C}$ homonuclear coupling among the directly bonded carbons can interfere with recoupling of the anisotropic interactions of interest. For CSA measurement, only a few recoupling pulse sequences, namely ROCSA [1] and $\mathrm{R} 18_{1}{ }^{7}[2-4]$, have been designed specifically for uniformly labeled samples by minimizing the effects from homonuclear dipolar interaction. In this work, we show that a simple method of CSA amplification can be applied directly to uniformly labeled systems. CSA amplification is a class of pulse sequences that uses $\pi$-pulses to repeatedly interrupt the averaging of CSA by MAS. The timing of pulses can be designed in a way such that the effective CSA evolution yields spinning sideband manifolds equivalent to those at a much lower spinning frequency. As shown in early works by Maricq and Waugh [5], and Herzfeld and Berger [6], spinning sidebands are a direct result from modulation of the CSA by MAS. For spinning rates that are several times smaller than the breadth of the anisotropy, the spectral intensity is concentrated into a handful of narrow spinning sidebands that can be fitted to determine the CSA tensor parameters. This 'slow spinning' method has become a method of choice for CSA measurement of dilute spin systems wherein CSA is the only spin interaction present and the line width is usually independent of the spinning frequency. For example, it has been possible to 
measure the ${ }^{15} \mathrm{~N}$ CSA in uniformly ${ }^{15} \mathrm{~N}$ labeled GB1 protein samples [7, 8], since homonuclear coupling among ${ }^{15} \mathrm{~N}$ nuclei is weak. Nevertheless, dipolar coupling among covalently bonded ${ }^{13} \mathrm{C}$ requires fast MAS to obtain high spectral resolution, making the slow spinning method unsuitable. The CSA amplification method presented here makes the measurement of spinning sideband intensities possible under fast MAS highresolution conditions.

In the following, a brief theory of our CSA amplification method [9] (dubbed $\mathrm{xCSA}$ ) is presented, as well as why homonuclear dipolar coupling does not affect it. This key feature, essential for application to uniformly ${ }^{13} \mathrm{C}$ labeled systems, is examined experimentally using an alanine sample. A comparison of ${ }^{13} \mathrm{C}$ sideband intensities of $\left[{ }^{13} \mathrm{C},{ }^{15} \mathrm{~N}\right]$ and $\left[{ }^{15} \mathrm{~N}\right]$ labeled alanine verifies that the homonuclear couplings have indeed negligible effects on the CSA measurement. For application to uniformly labeled proteins and macromolecules, the xCSA pulse sequence needs to be combined with twodimensional ${ }^{13} \mathrm{C} /{ }^{15} \mathrm{~N}$ correlation to achieve site resolution. Three-dimensional $\mathrm{xCSA}$ experiments with NCA and $\mathrm{NCO}$ correlation for ${ }^{13} \mathrm{C} /{ }^{15} \mathrm{~N}$ CSA measurement of the backbone $\mathrm{NH}, \mathrm{CA}$, and $\mathrm{CO}$ sites are demonstrated with a sample of $\left[\mathrm{U}-{ }^{13} \mathrm{C},{ }^{15} \mathrm{~N}\right]$ labeled GB1 protein.

\section{Theory}

CSA amplification is a class of pulse sequences for rotating solids pioneered by Griffin et al. [10] and Gullion [11], which utilizes $\pi$-pulses to interrupt the averaging of CSA under MAS. The timing of the $\pi$-pulses can be designed such that the effective evolution yields sidebands that are exactly the same as if the spinning rate is reduced or 
the CSA is magnified, hence these methods have been dubbed extended chemical shift modulation (XCS) or CSA amplification. The relative sideband intensities are the same when multiplying the anisotropy or reducing the spinning frequency by a factor $\kappa$. A number of CSA amplification pulse sequences have been developed to obtain the largest amplification factor, while using the minimum number of $\pi$-pulses and shortest total duration. A recent review on this topic provides a thorough explanation and comparison of these sequences [12]. In the following, we use our recent CSA amplification sequence [9], dubbed extended CSA amplification (xCSA), to describe the theory of CSA amplification and the effect from homonuclear dipolar couplings.

Under MAS, the chemical shift interaction can be separated into the constant isotropic shift and the modulating components from the CSA,

$$
\omega(t)=\omega_{i s o}+\omega_{C S A}(t), \quad \omega_{C S A}(t)=\sum_{m= \pm 1, \pm 2} \omega_{m} \exp \left(i m \omega_{r} t\right)
$$

The signal phase evolution can be obtained by integration of the modulated chemical shift over time $\omega_{C S A}(t)$, in terms of the indefinite integral $\xi(t)$,

$$
\phi_{C S A}=\int_{t_{a}}^{t_{b}} \omega_{C S A}(t) d t=\left.\xi(t)\right|_{t_{a}} ^{t_{b}}
$$

Application of $\pi$-pulses alternates the sign of the accumulated signal phase. At the end of a sequence of $n \pi$-pulses, the total phase becomes

$$
\phi_{\text {total }}=\left.\sum_{q=0}^{n}(-1)^{n+q} \xi(t)\right|_{t_{q}} ^{t_{q+1}}
$$

Fig. 1a shows our recent CSA amplification sequence designed based on the magic-angle turning (MAT) experiment $[13,14]$. The sequence consists of a basic amplification unit (Fig. 1b) with four $\pi$-pulses spanning two rotor periods. The $\pi$-pulses are separated by 
time units of $\tau_{r} / 3$, where $\tau_{r}\left(=1 / v_{r}\right)$ is the rotor period. The total evolution for the four evolution segments of the basic amplification unit can be written out explicitly

$$
\begin{aligned}
\phi_{\text {total }} & =\left[\xi\left(t_{0}+\frac{2 \tau_{r}}{3}\right)-\xi\left(t_{0}\right)\right]-\left[\xi\left(t_{0}\right)-\xi\left(t_{0}+\frac{2 \tau_{r}}{3}\right)\right] \\
& +\left[\xi\left(t_{0}+\frac{\tau_{r}}{3}\right)-\xi\left(t_{0}\right)\right]-\left[\xi\left(t_{0}\right)-\xi\left(t_{0}+\frac{\tau_{r}}{3}\right)\right] \\
& =-6 \xi\left(t_{0}\right)+2 \xi\left(t_{0}\right)+2 \xi\left(t_{0}+\frac{\tau_{r}}{3}\right)+2 \xi\left(t_{0}+\frac{2 \tau_{r}}{3}\right)
\end{aligned}
$$

Here $t_{0}$ is the time at the start of the pulse sequence. Invoking the MAT condition for a second-rank tensor $[9,13,14]$, such as CSA,

$$
\xi(t)+\xi\left(t+\frac{\tau_{r}}{3}\right)+\xi\left(t+\frac{2 \tau_{r}}{3}\right)=c
$$

where $c$ is a constant, Eqn. 4 becomes

$$
\phi_{\text {total }}=-6 \xi\left(t_{0}\right)+2 c
$$

The complete CSA amplification sequence consists of two basic amplification units. The second unit is shifted by a $t_{1}$ evolution time (Fig. 1a). The last $\pi$-pulse of the first basic unit is eliminated such that total phase evolution takes the form

$$
\phi_{x C S A}\left(t_{1}\right)=6\left[\xi\left(t_{0}\right)-\xi\left(t_{0}+t_{1}\right)\right]
$$

The result shows that the CSA evolution is amplified by a factor $\kappa=6$. In addition, it can be seen from the coherence transfer diagram in Fig. 1a that the total duration of the $p=$ +1 and -1 segments remain equal while $t_{1}$ is incremented. Hence, the isotropic shift is kept refocused while the CSA is amplified. Refocusing of the isotropic chemical shift is necessary to ensure that only the periodic modulation of the amplified CSA is present during $t_{1}$. Thus, Fourier transform of $t_{1}$ increments spanning one rotor period yields spinning sideband intensities corresponding to amplified CSA. More amplification units (Fig. 1b) can be inserted into the positions denoted by arrows in the pulse sequences of 
Fig. 1 to obtain larger amplification factors. In general, xCSA sequences consist of $(8 \cdot n-$

1) $\pi$-pulses covering $4 \cdot n$ rotor periods to yield amplification factors of $\kappa=6 \cdot n$.

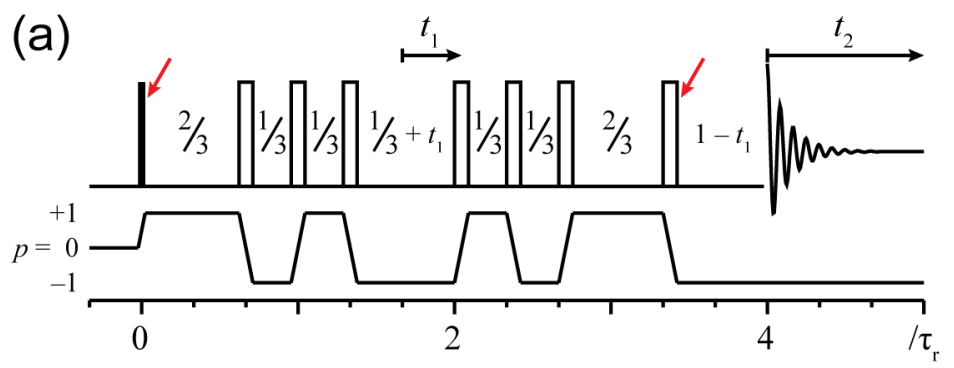

(b)

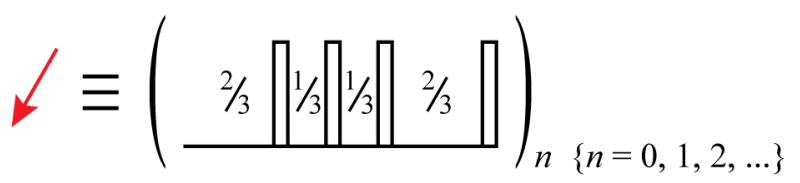

(c)

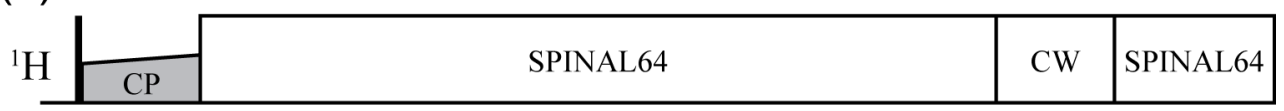
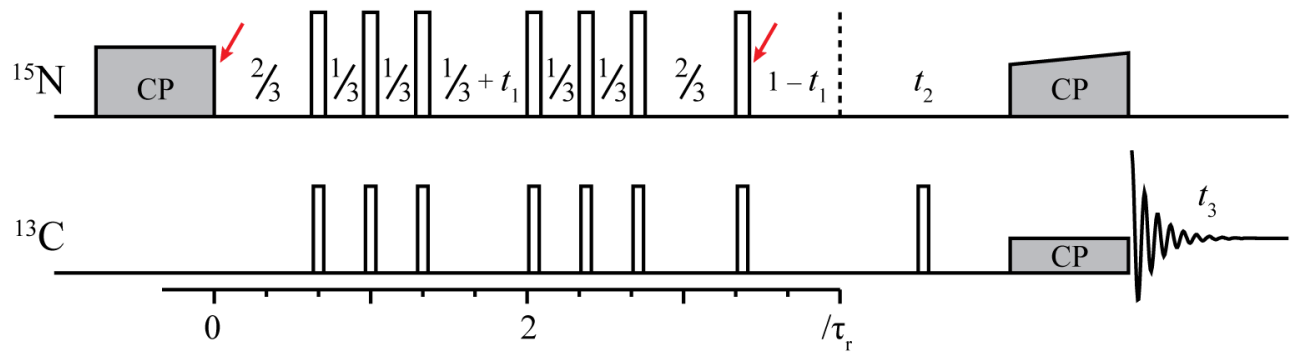

(d)
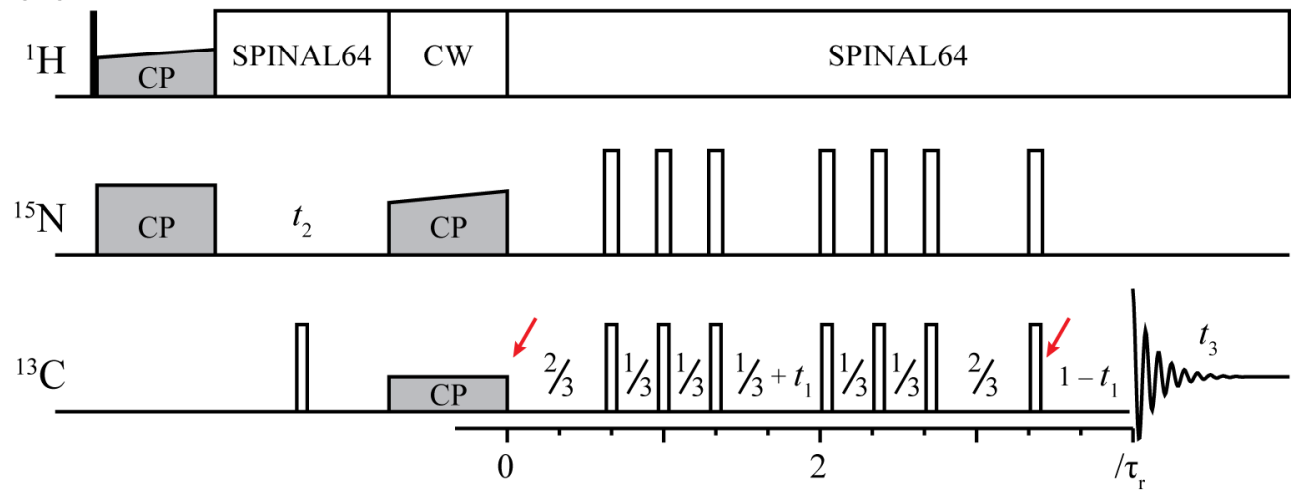

Fig. 1. (a) Pulse sequence and coherence transfer pathway for a $\kappa=6 x$ CSA experiment. (b) Basic amplification unit that can be inserted at the positions denoted by red arrows if additional CSA amplification is desired. (c, d) 3D xCSA pulse sequences for measurement of (c) ${ }^{15} \mathrm{~N}$ and (d) ${ }^{13} \mathrm{C} \mathrm{CSA}$. In (d), the ${ }^{15} \mathrm{~N} /{ }^{13} \mathrm{C} \mathrm{CP}$ and the ${ }^{13} \mathrm{C}$ carrier frequency can be changed to obtain NCA or NCO correlation. Narrow solid and empty rectangles in the pulse sequence denote $\pi / 2$ - and $\pi$-pulses, respectively. A cogwheel phase cycle is employed during the CSA amplification sequences to select the desired alternating 
coherence transfer pathway: 0 for all odd-numbered pulses starting from the excitation or CP pulse, $\{0,1$, $2, \ldots, 2 a+1\} \times \pi /(a+1)$ for all even-numbered pulses, and $\{0, \pi\}$ for the receiver phase, where $a$ is the total number of $\pi$-pulses.

For uniformly ${ }^{13} \mathrm{C}$ and ${ }^{15} \mathrm{~N}$ labeled samples, both homo- and hetero-nuclear interactions are present in addition to the CSA being measured. For homonuclear coupling, the effect of $\pi$-pulses can be described in a toggling frame. A $\pi$-pulse applied about the $x$-axis transforms the spin operators such that $I_{z} \rightarrow-I_{z}, I_{y} \rightarrow-I_{y}, I_{x} \rightarrow I_{x}$, effectively reversing the sign of the chemical shift Hamiltonian. However, the spin operators of the homonuclear dipolar coupling are bilinear and therefore their signs remain unchanged by the $\pi$-pulses throughout the whole xCSA sequence. At the end of the constant evolution time the homonuclear dipolar evolution becomes null due to the averaging of the dipolar coupling spatial components by MAS at complete rotor cycles. Hence, homonuclear dipolar coupling has no first-order effect on the CSA amplification. This is why xCSA can be applied directly to ${ }^{13} \mathrm{C}$ uniformly enriched systems without any modification. It should be mentioned that the homonuclear $J$-coupling remains constant under MAS leading to a signal attenuation $\cos (\pi J \tau)$ for a constant-time xCSA experiment of duration $\tau$. The short evolution time of xCSA usually makes this signal attenuation negligible. Most importantly, the constant attenuation does not introduce any $t_{1}$ modulation and therefore does not affect the CSA measurement. Similarly, heteronuclear dipolar coupling can be decoupled by applying $\pi$-pulses that are synchronous with the $\mathrm{xCSA} \pi$-pulses, as illustrated in the pulse sequences of Figs. 1c and $1 \mathrm{~d}$. In this way, the spin part of the heteronuclear dipolar interaction remains unchanged because synchronous $\pi$-pulses are applied to both nuclei and the fast MAS averages out the spatial component of the second-rank dipolar interaction. Again the heteronuclear scalar $J$ - 
coupling remains, but it is usually small for the constant evolution times typical used for the xCSA experiment.

With complete refocusing of isotropic shift and amplification of CSA in $t_{1}$, the 2D signal of the $\mathrm{xCSA}$ experiment can be generally expressed as

$$
\begin{aligned}
s\left(t_{1}, t_{2}\right) & =\left\langle e^{-i \phi_{x C S A}\left(t_{1}\right)} e^{-i \phi_{C S A}\left(t_{2}\right)-i \omega_{\text {iso }} t_{2}}\right\rangle \\
& =\left\langle e^{-i \kappa\left[\xi\left(t_{1}\right)-\xi(0)\right]} e^{-i\left[\xi\left(t_{2}\right)-\xi(0)\right]-i \omega_{\text {iso }} t_{2}}\right\rangle
\end{aligned}
$$

where \langle\rangle denotes a powder average. Under fast spinning, the CSA modulation during $t_{2}$ can be neglected and the time-domain data can be simplified as

$$
s\left(t_{1}, t_{2}\right)=\left\langle e^{-i \kappa\left[\xi\left(t_{1}\right)-\xi(0)\right]}\right\rangle e^{-i \omega_{i s o} t_{2}}
$$

Since $\xi(t)$ is cyclic with respect to the rotor period, $t_{1}$ increments spanning only one rotor period are sufficient to encode the complete CSA modulation/evolution. A Fourier transformation in $t_{1}$ yields a spinning sideband intensity profile corresponding to a CSA that has been scaled up by a factor $\kappa$, as illustrated in Fig. 2. It is evident from Eqn. 9 and Fig. 2 a that over one rotor period the real and imaginary parts of the $t_{1}$ time-domain data are symmetric and antisymmetric, respectively,

$$
s\left(t_{1}, f_{2}\right)=s^{*}\left(\tau_{r}-t_{1}, f_{2}\right)
$$

Fig. $2 \mathrm{~b}$ illustrates the modulation of $s\left(t_{1}, f_{2}\right)$ for the $\mathrm{CH}$ site of $\left[{ }^{15} \mathrm{~N}\right]$ alanine. Typically, phase-modulated signals from $t_{1}$-evolution of a single coherence transfer pathway is associated with phase-twisted line shapes [15]. In this case, though the $s\left(t_{1}, f_{2}\right)$ slices have mixed-phase (Fig. 2b), the final 2D spectrum $s\left(f_{1}, f_{2}\right)$ is completely absorptive (Fig. 2c). This is similar to the case of the phase-adjusted spinning sidebands (PASS) experiment [16-18]. Both xCSA and PASS have a periodic $t_{1}$-evolution that spans one rotor period. 
(a)
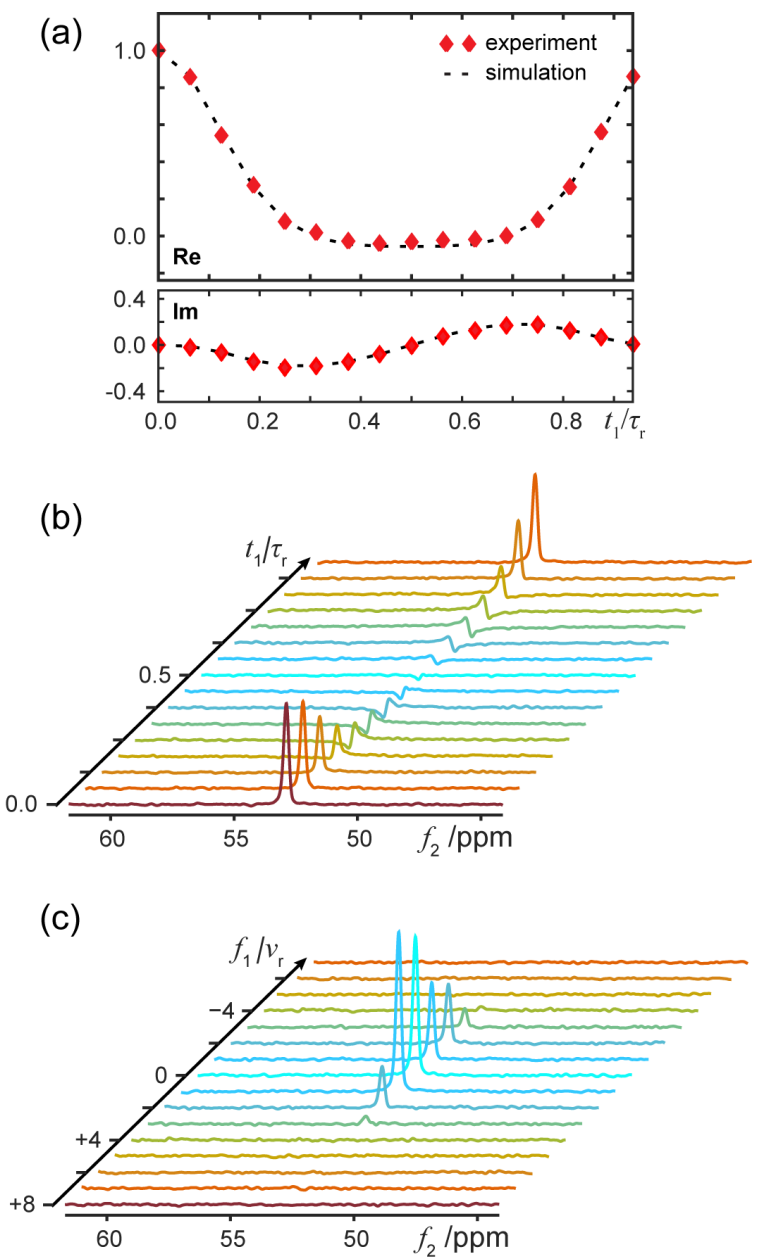

Fig. 2. (a) Plots of the real and imaginary parts of the $t_{1}$ time-domain signal for the $\mathrm{CH}$ peak of $\left[{ }^{15} \mathrm{~N}\right]$ alanine and stacked plots of the $s\left(t_{1}, f_{2}\right)(\mathrm{b})$ and $(\mathrm{c}) s\left(f_{1}, f_{2}\right)$ xCSA spectrum before and after $t_{1}$ Fourier transform.

Interpretation of $\mathrm{xCSA}$ as a direct amplification of CSA is only strictly valid as in Eqn. 9 when the sideband intensities in $f_{2}$ are negligible, or are all summed. For sites with large CSA, like protein backbone ${ }^{13} \mathrm{CO}$, there can be significant spinning sidebands in $f_{2}$. In practice, it is often easier and advantageous in terms of signal-to-noise to measure only the center-band peak intensity. Hence, it is necessary to derive the general expressions for the 2D xCSA spinning sideband intensities. The equations derived in the remainder of this section are used to perform numerical simulations and fit xCSA data by measuring 
only the centerband intensities. A MATLAB [19] script for the computation is given in the Appendix.

The indefinite integral $\xi(t)$ and Fourier expansion coefficients of $e^{i \xi(t)}$ and $e^{i \kappa \xi(t)}$ can be numerically computed quickly using a Fast Fourier Transformation (FFT)

$$
\begin{aligned}
& e^{i \xi(t)}=\sum_{k} a_{k} e^{i k \omega_{r} t} \\
& e^{i \kappa \xi(t)}=\sum_{k} b_{k} e^{i k \omega_{r} t}
\end{aligned}
$$

We can then express the general xCSA signal of Eqn. 8 as a Fourier series

$$
s\left(t_{1}, t_{2}\right) \propto\left\langle\sum_{k, l, m, n} b_{k}^{*} b_{l} a_{m}^{*} a_{n} e^{-i k \omega_{r} t_{1}} e^{-i m \omega_{r} t_{2}} e^{-i(k-l+m-n) \gamma}\right\rangle_{\alpha, \beta, \gamma} e^{-i \omega_{i s o} t_{2}}
$$

where \langle\rangle$_{\alpha, \beta, \gamma}$ denotes a powder average over the three Euler angles $(\alpha, \beta, \gamma)$ that rotate the molecular axis frame into the rotor frame. The average over the rotor angle $\gamma$ can be obtained by simply keeping only the terms with $k-l+m-n=0$, leading to the general expression for the 2D xCSA sideband intensities in terms of the Fourier coefficients,

$$
s(k, m) \propto\left\langle\sum_{l} b_{k}^{*} b_{l} a_{m}^{*} a_{k-l+m}\right\rangle_{\alpha, \beta}
$$

noting that the powder average is reduced to two polar angles $(\alpha, \beta)$. By setting $m=0$, we obtain the formula for calculating the $f_{1}$ spinning sideband manifold obtained by measuring only the intensities from the $f_{2}$ isotropic centerband

$$
s(k, 0) \propto\left\langle\sum_{l} b_{k}^{*} b_{l} a_{0}^{*} a_{k-l}\right\rangle_{\alpha, \beta}
$$

We can examine the case of fast spinning, where there are no spinning sidebands in $f_{2}$, i.e., $a_{0}=1$ and $a_{k-l}=0$ for $k \neq l$,

$$
s(k) \propto\left\langle\left|b_{k}\right|^{2}\right\rangle_{\alpha, \beta}
$$


Since $b_{k}$ are the Fourier expansion coefficients of $e^{i \kappa \xi(t)}$, the xCSA sideband intensities in $f_{1}$ are the same as the spinning sidebands of a regular 1D MAS spectrum with its CSA scaled up by factor $\kappa$. The main contribution to the sideband intensities in Eqn. 14 comes from terms with $k$ close to $l$. In the MATLAB script given in the Appendix, the variable ks defines how many of these terms are summed.

\section{Experimental}

The samples of $\left[{ }^{15} \mathrm{~N}\right]$ and $\left[{ }^{13} \mathrm{C},{ }^{15} \mathrm{~N}\right]$ labeled alanine were purchased from Cambridge Isotope Laboratories, Inc. (CIL) and used without further purification. $\left[\mathrm{U}-{ }^{13} \mathrm{C},{ }^{15} \mathrm{~N}\right] \mathrm{GB} 1$ was expressed in E.coli BL21- (DE3) cells that grow in minimal media $\left(1 \mathrm{~g} / \mathrm{L}^{15} \mathrm{NH}_{4} \mathrm{Cl}, 2 \mathrm{~g} / \mathrm{L}{ }^{13} \mathrm{C}\right.$ glucose), protein expression was induced with $1 \mathrm{mM}$ isopropyl$\beta$-D-thiogalactoside for 4 hours. Purification of protein was carried out by anion exchange on a Q-Sepharose FF column, followed by gel filtration on a Superdex75 column. Peak fractions were pooled and concentrated with Centriplus $3 \mathrm{kDa}$ MWCO filters, and the buffer thoroughly exchanged to $20 \mathrm{mM}$ sodium phosphate, $\mathrm{pH}$ 8.0. The GB1 solution was concentrated to $25 \mathrm{mg} / \mathrm{ml}$ and then a $2 \mathrm{ml}$ aliquot was combined with a (66.7\% MPD + 33.3\% 2-propanol) precipitation solution by vortex mixing in aliquots of 2, 2, 1 , and $1 \mathrm{ml}$ and then incubated at $4{ }^{\circ} \mathrm{C}$ for durations of $7 \mathrm{~min}, 7 \mathrm{~min}, 7 \mathrm{~min}$, and 18 hours after each successive addition. The resulting solution was centrifuged at $5000 \mathrm{rpm}$ for two minutes and the precipitated microcrystalline sample was transferred into the NMR rotor.

All NMR experiments were performed on a wide bore $600 \mathrm{MHz}$ Bruker Avance I spectrometer using a $3.2 \mathrm{~mm}$ low-E MAS probe [20] designed and built at the NHMFL. 
The $r f$ fields employed for $\left({ }^{13} \mathrm{C}\right.$ and $\left.{ }^{15} \mathrm{~N}\right) \pi$-pulses and ${ }^{1} \mathrm{H}$ heteronuclear decoupling are 50 and $100 \mathrm{kHz}$, respectively. Both ${ }^{13} \mathrm{C}$ and ${ }^{15} \mathrm{~N} \pi$-pulse lengths are $10 \mu \mathrm{s} .{ }^{13} \mathrm{C}$ chemical shift were externally referenced to tetramethylsilane (TMS) by setting the high-frequency resonance of solid adamantane to $\delta_{i s o}=38.48 \mathrm{ppm}$ [21]. In the acquisition of multidimensional xCSA spectra, the $t_{1}$ increments span exactly one rotor and $t_{1}$ Fourier transformation is applied without any line broadening or first-point scaling. The xCSA evolution dimension is acquired in a phase-modulated manner without hypercomplex phase cycling, while the ${ }^{15} \mathrm{~N} t_{2}$ dimension in the sequences of Figs. 1c and 1d was acquired using the method of States [22].

\section{Results and discussion}

We first examine experimentally the effect of homonuclear dipolar coupling to the CSA amplification sequence. Fig. 3 compares spinning sideband manifold intensities obtained with xCSA under $12 \mathrm{kHz}$ MAS between $\left[{ }^{13} \mathrm{C},{ }^{15} \mathrm{~N}\right]$ and $\left[{ }^{15} \mathrm{~N}\right]$ enriched alanine samples. The two samples have the same ${ }^{13} \mathrm{C} C S A$ and ${ }^{13} \mathrm{C}-{ }^{15} \mathrm{~N}$ heteronuclear coupling. The ${ }^{13} \mathrm{C}-{ }^{13} \mathrm{C}$ homonuclear couplings in the $\left[{ }^{13} \mathrm{C},{ }^{15} \mathrm{~N}\right]$ enriched sample are sizeable, whereas they can be neglected in the $\left[{ }^{15} \mathrm{~N}\right]$ enriched sample due to the low ${ }^{13} \mathrm{C}$ natural abundance. The experimental results show nearly identical xCSA spinning sideband manifolds for the three sites of alanine acquired with various amplification factors. The $\mathrm{CO}$ has the largest CSA and an amplification of $\kappa=6$ at $12 \mathrm{kHz}$ MAS generates sufficient spinning sidebands to measure its CSA tensor (Fig. 3b). Hodgkinson and Emsley [23] have studied the behavior of spinning sideband manifolds and their analysis for extracting CSA parameters. They have observed from direct one-dimensional 
measurements that too many sidebands can introduce greater errors in CSA fitting. Hence, the use of MAS frequencies that only generate a handful of sidebands, such as for the CO site with $\kappa=6$, is recommended for optimal CSA measurement. For the CA site, $\kappa=6$ amplification generates only small first-order sidebands (Fig. 3a). Larger amplification factors of $\kappa=12$ or 18 (Figs. 3c and 3d) are needed to obtain a sufficient number of sidebands to determine CSA parameters accurately. The methyl peak requires $\kappa=18$ to measure the smallest ${ }^{13} \mathrm{C} \mathrm{CSA}$ among the three sites (Fig. 3e). In all cases, the spectra of the $\left[{ }^{13} \mathrm{C},{ }^{15} \mathrm{~N}\right]$ and $\left[{ }^{15} \mathrm{~N}\right]$ enriched samples are nearly identical, indicating a negligible effect from ${ }^{13} \mathrm{C}-{ }^{13} \mathrm{C}$ homonuclear dipolar coupling in the $\left[{ }^{13} \mathrm{C},{ }^{15} \mathrm{~N}\right]$ sample. The xCSA results for both samples are also in excellent agreement with the slow spinning 1D MAS sideband profiles acquired with their corresponding spinning frequencies $v_{r} / \kappa$ for $\left[{ }^{15} \mathrm{~N}\right]$ alanine. 


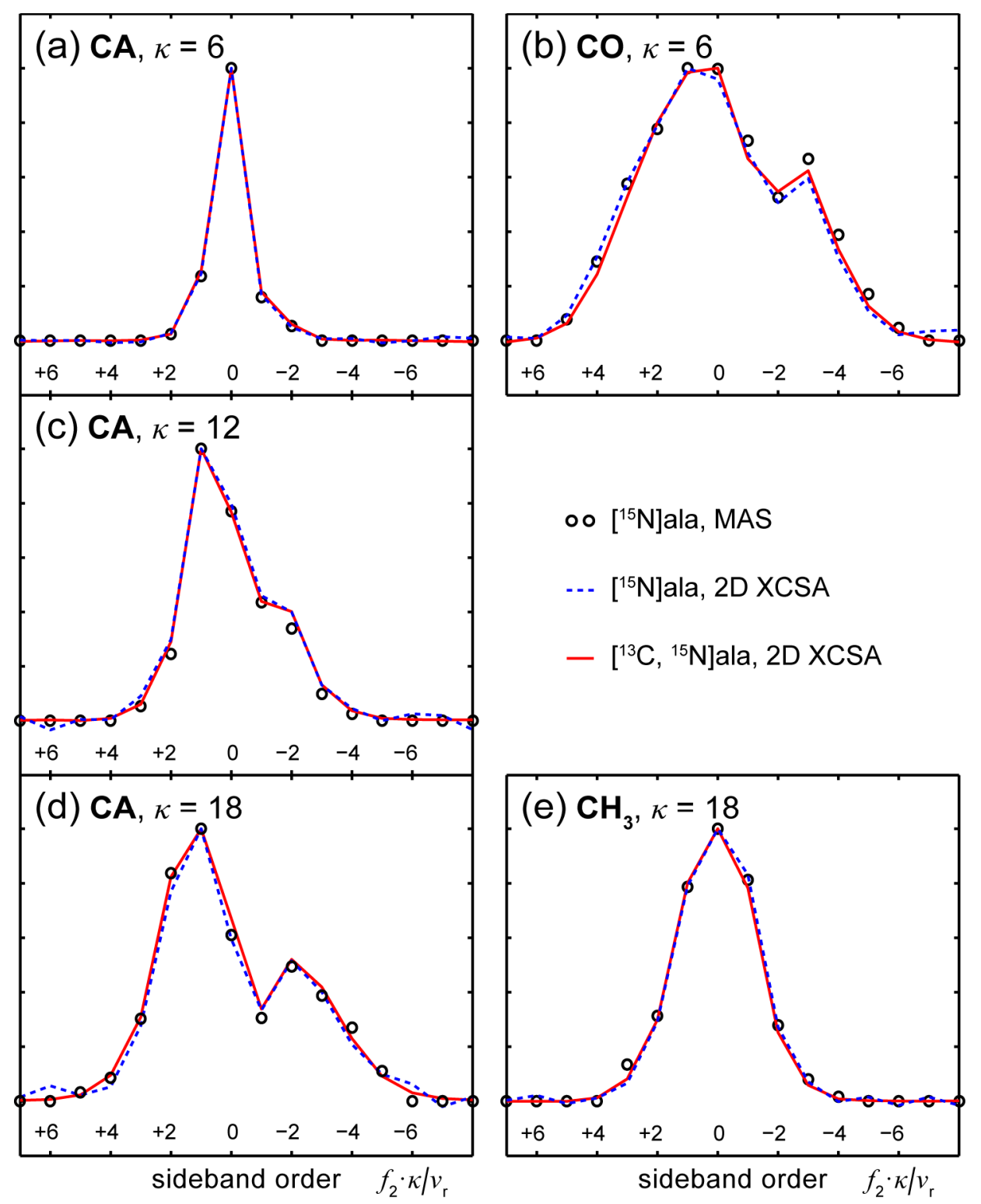

Fig. 3. Comparison of xCSA $f_{1}$ projections acquired at $v_{r}=12 \mathrm{kHz}$ with different $\kappa$ scaling factors for $\left[{ }^{15} \mathrm{~N}\right]$ alanine (blue dashes) and $\left[{ }^{13} \mathrm{C},{ }^{15} \mathrm{~N}\right]$ alanine (red lines) with $1 \mathrm{D}{ }^{13} \mathrm{C}$ slow MAS spectra of $\left[{ }^{15} \mathrm{~N}\right]$ alanine at the corresponding spinning frequencies $v_{r}=12 / \kappa \mathrm{kHz}$ (black circles).

For proteins, the isotropic shift resolution in a one-dimensional experiment is typically not sufficient to resolve overlap among the large number of sites. The 3D pulse sequences in Figs. $1 \mathrm{c}$ and $1 \mathrm{~d}$ combine $\mathrm{xCSA}$ with commonly used $2 \mathrm{D}{ }^{15} \mathrm{~N} /{ }^{13} \mathrm{C}$ correlation experiments, which in this instance provide near-complete site resolution for the GB1 protein sample. Fig. 4 illustrates the extraction of ${ }^{15} \mathrm{~N} /{ }^{13} \mathrm{C} \times \mathrm{CSA}$ sideband intensities from 
three 3D experiments for the three backbone CO-NH-CA sites of the amide bond between residues A48-T49. Amplification of $\kappa=6$ was used for the ${ }^{15} \mathrm{NH}$ and ${ }^{13} \mathrm{CO}$ sites, while $\kappa=12$ was used for the ${ }^{13} \mathrm{CA}$ sites. For each site, the xCSA sideband intensity profiles are fitted by comparing with numerical simulations using the centerband only formula in Eqn. 14 and the MATLAB script in the Appendix. The Appendix also shows the corresponding two-dimensional root-mean-square difference between the experimental and simulated sideband intensities as a function of the CSA fitting parameters. All fitted CSA parameters are tabulated in Table 1. It should be noted that the GB1 sample used here was prepared at $\mathrm{pH} 8.0$, which differs from the $\mathrm{pH} 5.5$ samples used in the literature for CSA measurement [24]. The ${ }^{15} \mathrm{NH},{ }^{13} \mathrm{CA}$ and ${ }^{13} \mathrm{CO}$ isotropic shifts for some of the residues have significant deviation with reported values. Hence, 3D NCACX and NCOCX spectra (not shown) were acquired and used to assign all backbone resonances.
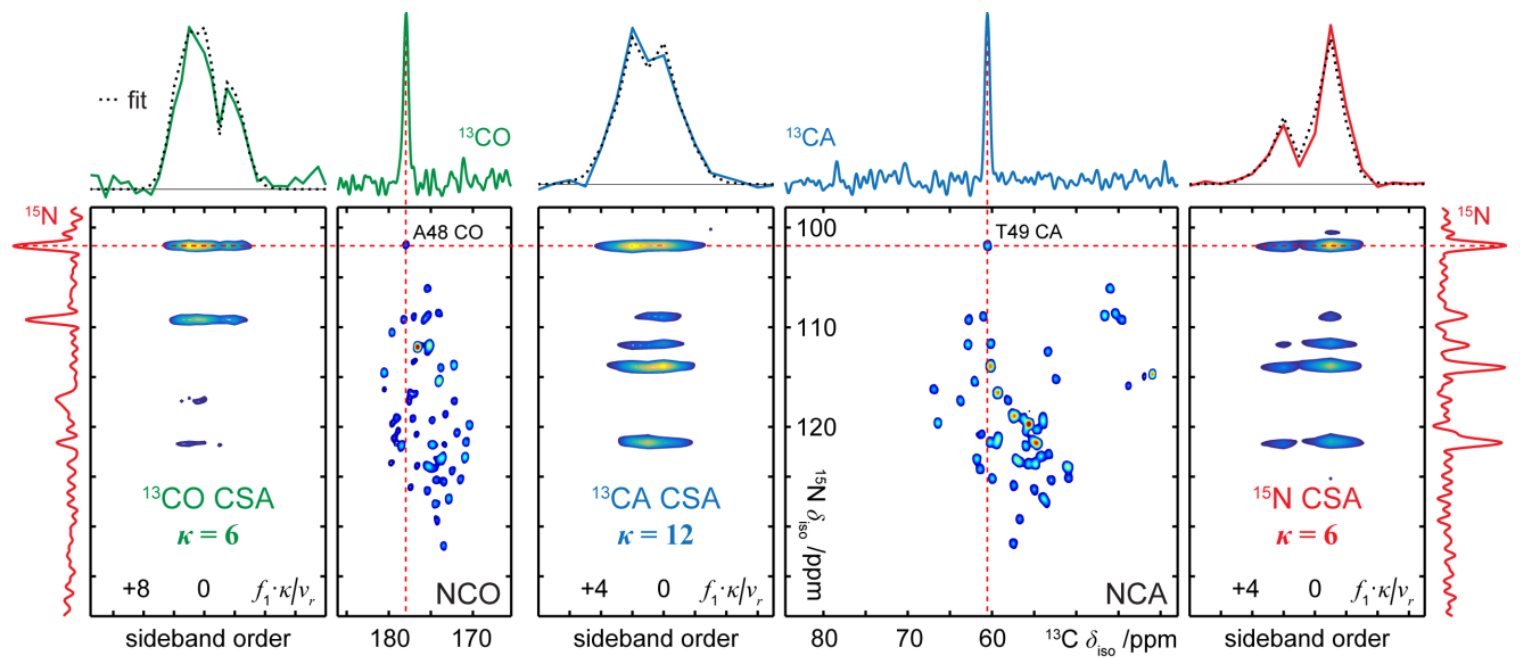

Fig. 4. Representative ${ }^{13} \mathrm{CO},{ }^{13} \mathrm{CA}$ and ${ }^{15} \mathrm{NH} \mathrm{xCSA}$ sideband manifold patterns obtained from $3 \mathrm{D}$ $\mathrm{NCO} / \mathrm{xCSA}\left({ }^{13} \mathrm{CO}\right), \mathrm{NCA} / \mathrm{xCSA}\left({ }^{13} \mathrm{CA}\right)$ and $\mathrm{NCA} / \mathrm{xCSA}\left({ }^{15} \mathrm{NH}\right)$ spectra, respectively, using the pulse sequences in Figs. 1c and 1d. All experiments were performed at $14.1 \mathrm{~T}$ with $10 \mathrm{kHz}$ MAS, $0.5 \mathrm{~s}$ recycle delay, and a maximum ${ }^{15} \mathrm{~N} t_{2}$ evolution time of $16 \mathrm{~ms}$. The amplification factor, number of xCSA $t_{1}$ points, scans and total experimental time for each experiment are: $\left({ }^{13} \mathrm{CO}: 6,32,32,11.4\right.$ hours $),\left({ }^{13} \mathrm{CA}: 12,16,64\right.$, 11.4 hours), $\left({ }^{15} \mathrm{NH}: 6,16,32,5.7\right.$ hours $)$. 
Fig. 5 shows plots of the three principal CSA components $\left(\delta_{11}, \delta_{22}, \delta_{33}\right)$ measured using xCSA for the GB1 backbone ${ }^{13} \mathrm{CA},{ }^{13} \mathrm{CO}$ and ${ }^{15} \mathrm{NH}$ sites. CSA parameters of GB1 have been measured previously using slow spinning and ROCSA methods $[8,25,26]$ and are also plotted in Fig. 5. The comparison shows good agreement with reported values. The ${ }^{13} \mathrm{CA}$ CSA shows significant variation through the protein sequence. In addition to the difference in residue type, the ${ }^{13} \mathrm{CA}$ CSA is also sensitive to peptide dihedral angles and protein secondary structure [27-29], and has been used for structure refinement [25]. The ${ }^{13} \mathrm{CO}$ and ${ }^{15} \mathrm{NH}$ CSA tensor components show less variation relative to their span. Since they are less sensitive to protein secondary structure, ${ }^{13} \mathrm{CO}$ and ${ }^{15} \mathrm{NH}$ CSA are often used to probe protein dynamics. In the present case, minimal scaling (due to dynamics) of the ${ }^{13} \mathrm{CO}$ and ${ }^{15} \mathrm{NH}$ CSA is observed since GB1 is a fairly rigid protein, as shown by the $\sim 0.95$ order parameters reported for most sites [26]. 

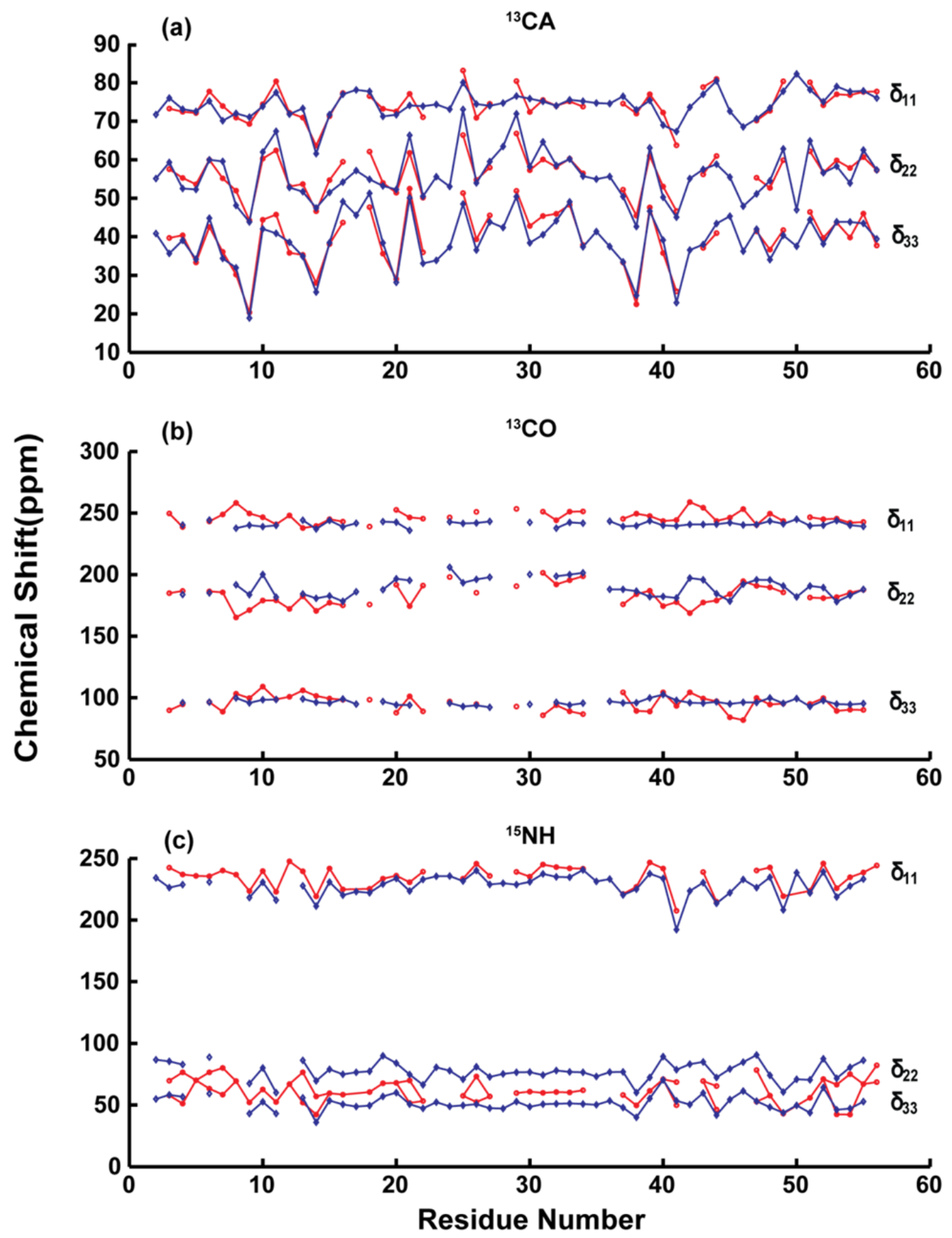

Fig. 5. Comparison of the GB1 backbone (a) ${ }^{13} \mathrm{CA}$, (b) ${ }^{13} \mathrm{CO}$ and (c) ${ }^{15} \mathrm{NH}$ CSA tensors determined in this work using $\mathrm{xCSA}$ (red traces) with values reported in the literature (blue traces).

The constant-time xCSA experiment has the advantage of not requiring line broadening when fitting spinning sideband intensities, as with ROCSA, to extract CSA 
tensor parameters. It has been noted in the literature that line broadening and CSA parameters are correlated when fitting CSA powder patterns [7]. For example, the CSA tensors of amide ${ }^{15} \mathrm{NH}$ sites usually have asymmetry factors near to zero (i.e., $\delta_{22} \approx \delta_{33}$ ), and applying line broadening tends to make the CSA patterns appear to have a larger difference between $\delta_{22}$ and $\delta_{33}$. This may lead to the tendency to smaller differences between $\delta_{22}$ and $\delta_{33}$ for amide ${ }^{15} \mathrm{NH}$ sites measured by xCSA (Fig. 5c) than with experiments such as ROCSA.

Non-ideal $\pi$-pulses arising from inhomogeneous $r f$ fields, frequency offset and finite pulse lengths can lead to signals of coherence transfer pathways other than the one shown in Fig. 1a. The employed cogwheel phase cycle removes these signals, ensuring the acquisition of only the desired alternating pathway. As a result, distortions due to non-ideal $\pi$-pulses are avoided by the filtering of undesired signals. However, the efficiency of the xCSA experiment decreases, especially when a large number of $\pi$-pulses are needed for large amplification factors. For example, the efficiencies of the xCSA experiments in Figs. 3a ( $\kappa=6,7 \times \pi$-pulses), $3 \mathrm{c}(\kappa=12,15 \times \pi$-pulses $)$ and $3 \mathrm{~d}(\kappa=6$, $23 \times \pi$-pulses) are approximately 80,70 and $60 \%$, respectively, compared to a conventional CP/MAS spectrum.

\section{Conclusions}

In summary, CSA amplification by xCSA is a robust method for measuring ${ }^{13} \mathrm{C} /{ }^{15} \mathrm{~N}$ CSA of uniformly ${ }^{13} \mathrm{C}$ and ${ }^{15} \mathrm{~N}$ enriched samples. Both theory and experimental results show that ${ }^{13} \mathrm{C}$ homonuclear dipolar coupling does not interfere with $\mathrm{xCSA}$ amplification. With xCSA, the amplification factor can be varied depending on the 
applied spinning speed and magnitude of the CSA to obtain the optimal number of spinning sidebands for measuring CSA tensors accurately. The xCSA pulse sequence uses a short constant evolution-time of $4 \cdot n$ rotor periods and $(8 \cdot n-1) \pi$-pulses to achieve amplification factors of $\kappa=6 \cdot n$. The amplified spinning sideband manifolds can be fitted in a straight forward manner without the implications of line broadening and scaling factors present in other recoupling methods. As illustrated with a model protein sample, the xCSA experiment can be combined with multi-dimensional experiments to measure ${ }^{13} \mathrm{C} /{ }^{15} \mathrm{~N}$ CSA parameters of proteins and provide valuable information of their structure and dynamics.

Table 1 Isotropic shifts ${ }^{\mathrm{a}}$ and CSA tensor elements ${ }^{\mathrm{b}}$ determined in this study for the GB1 backbone ${ }^{15} \mathrm{NH}$, ${ }^{13} \mathrm{CA}$ and ${ }^{13} \mathrm{CO}$ sites.

\begin{tabular}{|c|c|c|c|c|c|c|c|c|c|c|c|c|}
\hline \multirow[b]{2}{*}{ residue } & \multicolumn{4}{|c|}{${ }^{15} \mathrm{NH}$} & \multicolumn{4}{|c|}{${ }^{13} \mathrm{CA}$} & \multicolumn{4}{|c|}{${ }^{13} \mathrm{CO}$} \\
\hline & $\begin{array}{c}\delta_{11} \\
(\mathrm{ppm})\end{array}$ & $\begin{array}{c}\delta_{22} \\
(\mathrm{ppm})\end{array}$ & $\begin{array}{c}\delta_{33} \\
(\mathrm{ppm})\end{array}$ & $\begin{array}{c}\delta_{i s o} \\
(\mathrm{ppm})\end{array}$ & $\begin{array}{c}\delta_{11} \\
(\mathrm{ppm})\end{array}$ & $\begin{array}{c}\delta_{22} \\
(\mathrm{ppm})\end{array}$ & $\begin{array}{c}\delta_{33} \\
(\mathrm{ppm})\end{array}$ & $\begin{array}{c}\delta_{i s o} \\
(\mathrm{ppm})\end{array}$ & $\begin{array}{c}\delta_{11} \\
(\mathrm{ppm})\end{array}$ & $\begin{array}{c}\delta_{22} \\
(\mathrm{ppm})\end{array}$ & $\begin{array}{c}\delta_{33} \\
(\mathrm{ppm})\end{array}$ & $\begin{array}{c}\delta_{\text {iso }} \\
(\mathrm{ppm})\end{array}$ \\
\hline Y3 & 242 & 70 & 58 & 123.3 & 73 & 58 & 40 & 56.8 & 250 & 185 & 90 & 174.8 \\
\hline K4 & 237 & 77 & 51 & 121.6 & 72 & 55 & 40 & 56.0 & 239 & 187 & 95 & 173.4 \\
\hline L5 & 236 & 70 & 70 & 125.3 & 72 & 54 & 33 & 53.0 & & & & \\
\hline I6 & 236 & 77 & 63 & 125.2 & 78 & 60 & 43 & 60.1 & 243 & 186 & 97 & 175.4 \\
\hline I7 & 240 & 80 & 58 & 126.2 & 74 & 55 & 36 & 55.1 & 249 & 186 & 89 & 174.4 \\
\hline N8 & 237 & 69 & 69 & 125.2 & 71 & 52 & 30 & 51.0 & 258 & 165 & 103 & 175.6 \\
\hline G9 & 224 & 52 & 52 & 109.3 & 69 & 44 & 20 & 44.6 & 250 & 171 & 100 & 173.5 \\
\hline K10 & 240 & 63 & 63 & 121.7 & 74 & 60 & 44 & 59.7 & 247 & 179 & 109 & 178.2 \\
\hline $\mathrm{T} 11$ & 223 & 53 & 52 & 109.3 & 80 & 62 & 46 & 62.8 & 241 & 179 & 99 & 172.9 \\
\hline L12 & 248 & 67 & 67 & 127.2 & 72 & 53 & 36 & 53.7 & 248 & 172 & 101 & 173.7 \\
\hline K13 & 240 & 77 & 52 & 122.7 & 71 & 54 & 35 & 53.3 & 238 & 182 & 106 & 175.4 \\
\hline G14 & 219 & 57 & 42 & 106.2 & 64 & 47 & 28 & 46.1 & 239 & 171 & 102 & 170.5 \\
\hline E15 & 242 & 60 & 60 & 120.3 & 71 & 55 & 38 & 54.7 & 245 & 177 & 99 & 173.8 \\
\hline T16 & 225 & 59 & 58 & 113.9 & 77 & 59 & 44 & 60.2 & 243 & 175 & 98 & 172.2 \\
\hline T18 & 225 & 61 & 61 & 115.5 & 77 & 62 & 48 & 62.1 & 239 & 176 & 98 & 171.0 \\
\hline E19 & 234 & 68 & 68 & 122.9 & 73 & 54 & 36 & 54.3 & & & & \\
\hline A20 & 236 & 68 & 68 & 124.0 & 73 & 51 & 29 & 51.0 & 253 & 192 & 88 & 177.4 \\
\hline V21 & 231 & 70 & 52 & 117.4 & 77 & 62 & 52 & 63.8 & 246 & 174 & 101 & 174.0 \\
\hline D22 & 239 & 53 & 53 & 115.2 & 71 & 50 & 36 & 52.4 & 246 & 191 & 89 & 175.2 \\
\hline A24 & & & & & & & & & 247 & 198 & 97 & 180.5 \\
\hline $\mathrm{T} 25$ & 234 & 58 & 58 & 116.2 & 83 & 66 & 51 & 67.0 & & & & \\
\hline A26 & 246 & 73 & 52 & 123.7 & 71 & 55 & 39 & 55.0 & 251 & 185 & 95 & 177.0 \\
\hline E27 & 236 & 57 & 57 & 116.6 & 75 & 58 & 46 & 59.4 & & & & \\
\hline V29 & 239 & 60 & 80 & 119.6 & 80 & 67 & 52 & 66.4 & 253 & 191 & 93 & 179.0 \\
\hline F30 & 235 & 61 & 61 & 118.9 & 72 & 57 & 43 & 57.5 & & & & \\
\hline K31 & 245 & 60 & 60 & 121.6 & 75 & 60 & 45 & 60.3 & 251 & 201 & 86 & 179.4 \\
\hline Q32 & 243 & 61 & 61 & 121.3 & 74 & 58 & 46 & 59.4 & 244 & 192 & 94 & 176.7 \\
\hline Y33 & 242 & 60 & 60 & 120.8 & 75 & 60 & 48 & 61.2 & 251 & 196 & 89 & 178.5 \\
\hline A34 & 242 & 62 & 62 & 121.9 & 74 & 56 & 38 & 56.0 & 251 & 199 & 87 & 178.9 \\
\hline N37 & 221 & 58 & 58 & 112.4 & 75 & 52 & 33 & 53.4 & 245 & 176 & 104 & 175.2 \\
\hline
\end{tabular}




\begin{tabular}{|c|c|c|c|c|c|c|c|c|c|c|c|c|}
\hline G38 & 227 & 50 & 50 & 108.8 & 72 & 46 & 22 & 46.7 & 250 & 184 & 89 & 174.3 \\
\hline V39 & 247 & 62 & 62 & 123.3 & 77 & 61 & 48 & 61.9 & 248 & 187 & 89 & 174.4 \\
\hline D40 & 242 & 71 & 71 & 127.8 & 72 & 53 & 36 & 53.6 & 243 & 174 & 104 & 174.1 \\
\hline G41 & 208 & 69 & 50 & 108.6 & 64 & 47 & 26 & 45.4 & 244 & 178 & 93 & 171.8 \\
\hline E42 & & & & & & & & & 259 & 169 & 104 & 177.4 \\
\hline W43 & 239 & 69 & 69 & 125.9 & 79 & 56 & 37 & 57.4 & 254 & 177 & 99 & 177.0 \\
\hline T44 & 215 & 65 & 46 & 108.9 & 81 & 61 & 41 & 61.0 & 244 & 179 & 97 & 173.2 \\
\hline Y45 & & & & & & & & & 246 & 184 & 84 & 171.5 \\
\hline D46 & & & & & & & & & 253 & 195 & 82 & 176.6 \\
\hline D47 & 240 & 78 & 53 & 123.7 & 70 & 55 & 41 & 55.6 & 240 & 191 & 100 & 177.1 \\
\hline A48 & 243 & 58 & 58 & 119.3 & 73 & 53 & 37 & 54.0 & 250 & 190 & 95 & 177.9 \\
\hline T49 & 219 & 43 & 43 & 101.8 & 80 & 60 & 42 & 60.7 & 244 & 186 & 95 & 174.8 \\
\hline T51 & 224 & 56 & 56 & 111.8 & 80 & 62 & 46 & 62.9 & 247 & 181 & 95 & 174.3 \\
\hline F52 & 246 & 71 & 71 & 129.3 & 74 & 57 & 40 & 56.8 & 245 & 181 & 100 & 175.2 \\
\hline T53 & 226 & 67 & 43 & 111.6 & 77 & 60 & 44 & 60.2 & 245 & 182 & 89 & 172.2 \\
\hline V54 & 235 & 75 & 42 & 117.3 & 77 & 58 & 40 & 58.1 & 242 & 185 & 90 & 172.5 \\
\hline T55 & 239 & 67 & 67 & 124.2 & 78 & 61 & 46 & 61.5 & 243 & 188 & 90 & 173.5 \\
\hline E56 & 244 & 82 & 69 & 131.7 & 78 & 57 & 38 & 57.6 & & & & \\
\hline Y3 & 242 & 70 & 58 & 123.3 & 73 & 58 & 40 & 56.8 & 250 & 185 & 90 & 174.8 \\
\hline
\end{tabular}

a the isotropic shift is defined as $\delta_{\text {iso }}=\left(\delta_{11}+\delta_{22}+\delta_{33}\right) / 3$

${ }^{\mathrm{b}}$ the CSA tensor component are defined such that $\delta_{11} \geq \delta_{22} \geq \delta_{33}$

\section{Acknowledgements}

This work was supported by the National High Magnetic Field Laboratory through NSF DMR-1157490, the State of Florida, the Ministry of Science and Technology grant 2013CB910200, and the 1000 Young Talents Program of China.

\section{References}

[1] J.C.C. Chan, R. Tycko, Recoupling of chemical shift anisotropies in solid-state NMR under high-speed magic-angle spinning and in uniformly C-13-labeled systems, J. Chem. Phys. 118 (2003) 8378-8389.

[2] X. Zhao, M. Eden, M.H. Levitt, Recoupling of heteronuclear dipolar interactions in solid-state NMR using symmetry-based pulse sequences, Chem. Phys. Lett. 342 (2001) 353-361.

[3] Y. Nishiyama, T. Yamazaki, T. Terao, Development of modulated rf sequences for decoupling and recoupling of nuclear-spin interactions in sample-spinning solidstate NMR: Application to chemical-shift anisotropy determination, J. Chem. Phys. 124 (2006) 064304.

[4] G. Hou, S. Paramasivam, I.J.L. Byeon, A.M. Gronenborn, T. Polenova, Determination of relative tensor orientations by gamma-encoded chemical shift anisotropy/heteronuclear dipolar coupling 3D NMR spectroscopy in biological solids, Phys. Chem. Chem. Phys. 12 (2010) 14873-14883.

[5] M.M. Maricq, J.S. Waugh, NMR in Rotating Solids, J. Chem. Phys. 70 (1979) 3300-3316. 
[6] J. Herzfeld, A.E. Berger, Sideband Intensities in NMR-Spectra of Samples Spinning at the Magic Angle, J. Chem. Phys. 73 (1980) 6021-6030.

[7] B.J. Wylie, W.T. Franks, C.M. Rienstra, Determinations of N-15 chemical shift anisotropy magnitudes in a uniformly N-15, C-13-labeled microcrystalline protein by three-dimensional magic-angle spinning nuclear magnetic resonance spectroscopy, J. Phys. Chem. B 110 (2006) 10926-10936.

[8] B.J. Wylie, L.J. Sperling, H.L. Frericks, G.J. Shah, W.T. Franks, C.M. Rienstra, Chemical-shift anisotropy measurements of amide and carbonyl resonances in a microcrystalline protein with slow magic-angle spinning NMR spectroscopy, J. Am. Chem. Soc. 129 (2007) 5318-5319.

[9] I. Hung, Z. Gan, An efficient amplification pulse sequence for measuring chemical shift anisotropy under fast magic-angle spinning, J. Magn. Reson. 213 (2011) 196199.

[10] D.P. Raleigh, A.C. Kolbert, T.G. Oas, M.H. Levitt, R.G. Griffin, Enhancement of the Effect of Small Anisotropies in Magic-Angle Spinning Nuclear MagneticResonance, J. Chem. Soc. Farad. T. 184 (1988) 3691-3711.

[11] T. Gullion, Extended Chemical-Shift Modulation, J. Magn. Reson. 85 (1989) 614619.

[12] L. Shao, J.J. Titman, Chemical shift anisotropy amplification, Prog. Nucl. Magn. Reson. Spectrosc. 51 (2007) 103-137.

[13] Z.H. Gan, High-Resolution Chemical-Shift and Chemical-Shift Anisotropy Correlation in Solids Using Slow Magic Angle Spinning, J. Am. Chem. Soc. 114 (1992) 8307-8309.

[14] J.Z. Hu, D.W. Alderman, C.H. Ye, R.J. Pugmire, D.M. Grant, An Isotropic Chemical Shift-Chemical Shift Anisotropy Magic-Angle Slow-Spinning 2d NMR Experiment, J. Magn. Reson. Ser. A 105 (1993) 82-87.

[15] R.R. Ernst, G. Bodenhausen, A. Wokaun, Principles of Nuclear Magnetic Resonance in One and Two Dimensions, Oxford University Press, New York, 1987.

[16] W.T. Dixon, Spinning-Sideband-Free and Spinning-Sideband-Only NMR-Spectra in Spinning Samples, J. Chem. Phys. 77 (1982) 1800-1809.

[17] O.N. Antzutkin, S.C. Shekar, M.H. Levitt, 2-Dimensional Side-Band Separation in Magic-Angle-Spinning NMR, J. Magn. Reson. Ser. A 115 (1995) 7-19.

[18] I. Hung, Z. Gan, On the magic-angle turning and phase-adjusted spinning sidebands experiments, J. Magn. Reson. 204 (2010) 150-154.

[19] MATLAB, version 8.4.0.150421 (R2014b), The MathWorks Inc., Natick, Massachusetts, 2014.

[20] S.A. McNeill, P.L. Gor'kov, K. Shetty, W.W. Brey, J.R. Long, A low-E magic angle spinning probe for biological solid state NMR at $750 \mathrm{MHz}$, J. Magn. Reson. 197 (2009) 135-144.

[21] C.R. Morcombe, K.W. Zilm, Chemical shift referencing in MAS solid state NMR, J. Magn. Reson. 162 (2003) 479-486.

[22] D.J. States, R.A. Haberkorn, D.J. Ruben, A Two-Dimensional Nuclear Overhauser Experiment with Pure Absorption Phase in 4 Quadrants, J. Magn. Reson. 48 (1982) 286-292.

[23] P. Hodgkinson, L. Emsley, The reliability of the determination of tensor parameters by solid-state nuclear magnetic resonance, J. Chem. Phys. 107 (1997) 4808-4816. 
[24] W.T. Franks, D.H. Zhou, B.J. Wylie, B.G. Money, D.T. Graesser, H.L. Frericks, G. Sahota, C.M. Rienstra, Magic-angle spinning solid-state NMR spectroscopy of the beta 1 immunoglobulin binding domain of protein $\mathrm{G}(\mathrm{GB} 1)$ : N-15 and C-13 chemical shift assignments and conformational analysis, J. Am. Chem. Soc. 127 (2005) 12291-12305.

[25] B.J. Wylie, C.D. Schwieters, E. Oldfield, C.M. Rienstra, Protein Structure Refinement Using C-13 alpha Chemical Shift Tensors, J. Am. Chem. Soc. 131 (2009) 985-992.

[26] B.J. Wylie, L.J. Sperling, A.J. Nieuwkoop, W.T. Franks, E. Oldfield, C.M. Rienstra, Ultrahigh resolution protein structures using NMR chemical shift tensors, Proc. Natl. Acad. Sci. U.S.A. 108 (2011) 16974-16979.

[27] J. Heller, D.D. Laws, M. Tomaselli, D.S. King, D.E. Wemmer, A. Pines, R.H. Havlin, E. Oldfield, Determination of dihedral angles in peptides through experimental and theoretical studies of alpha-carbon chemical shielding tensors, J. Am. Chem. Soc. 119 (1997) 7827-7831.

[28] M. Hong, Solid-state NMR determination of C-13 alpha chemical shift anisotropies for the identification of protein secondary structure, J. Am. Chem. Soc. 122 (2000) 3762-3770.

[29] E.Y. Chekmenev, R.Z. Xu, M.S. Mashuta, R.J. Wittebort, Glycyl C alpha chemical shielding in tripeptides: Measurement by solid-state NMR and correlation with Xray structure and theory, J. Am. Chem. Soc. 124 (2002) 11894-11899.

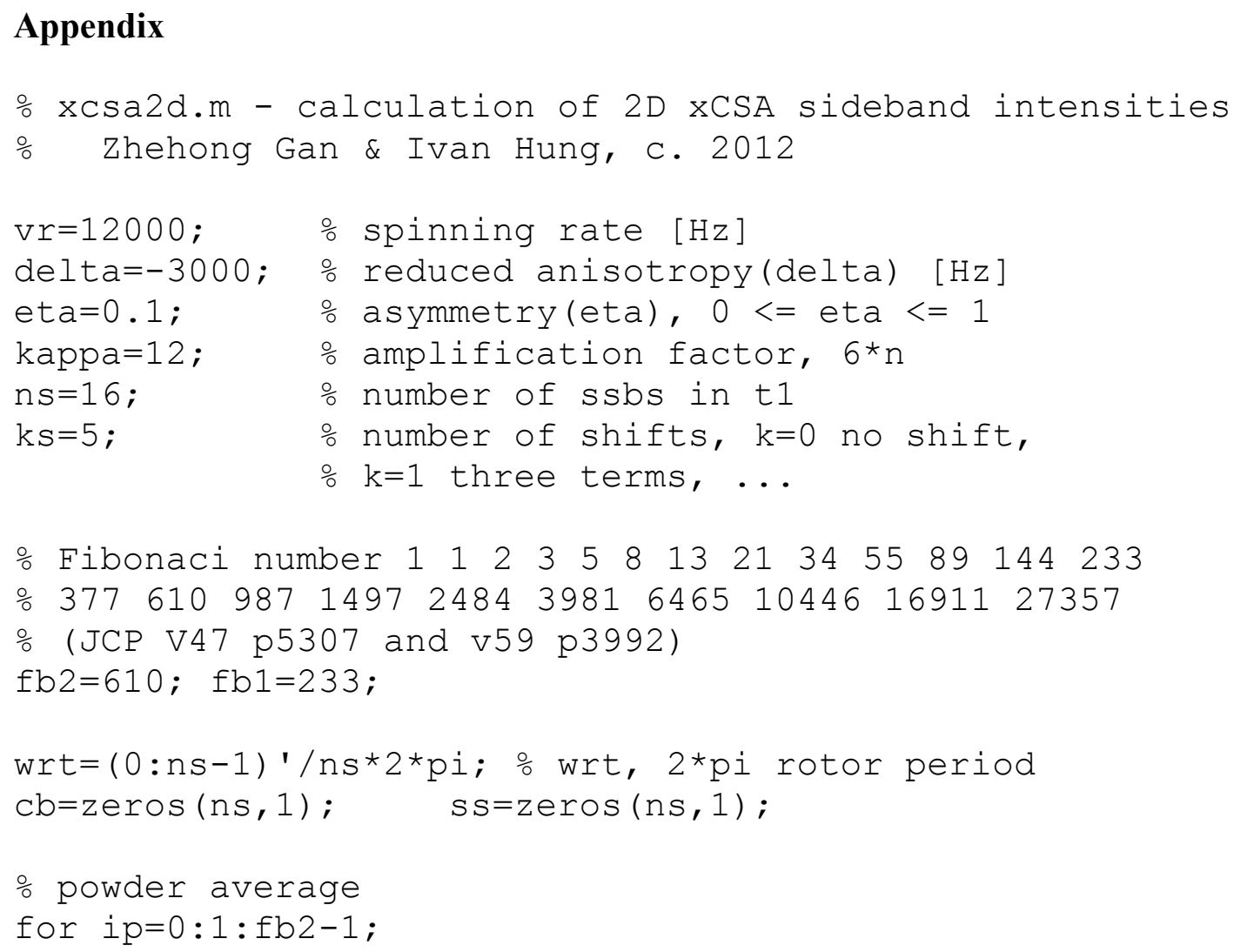




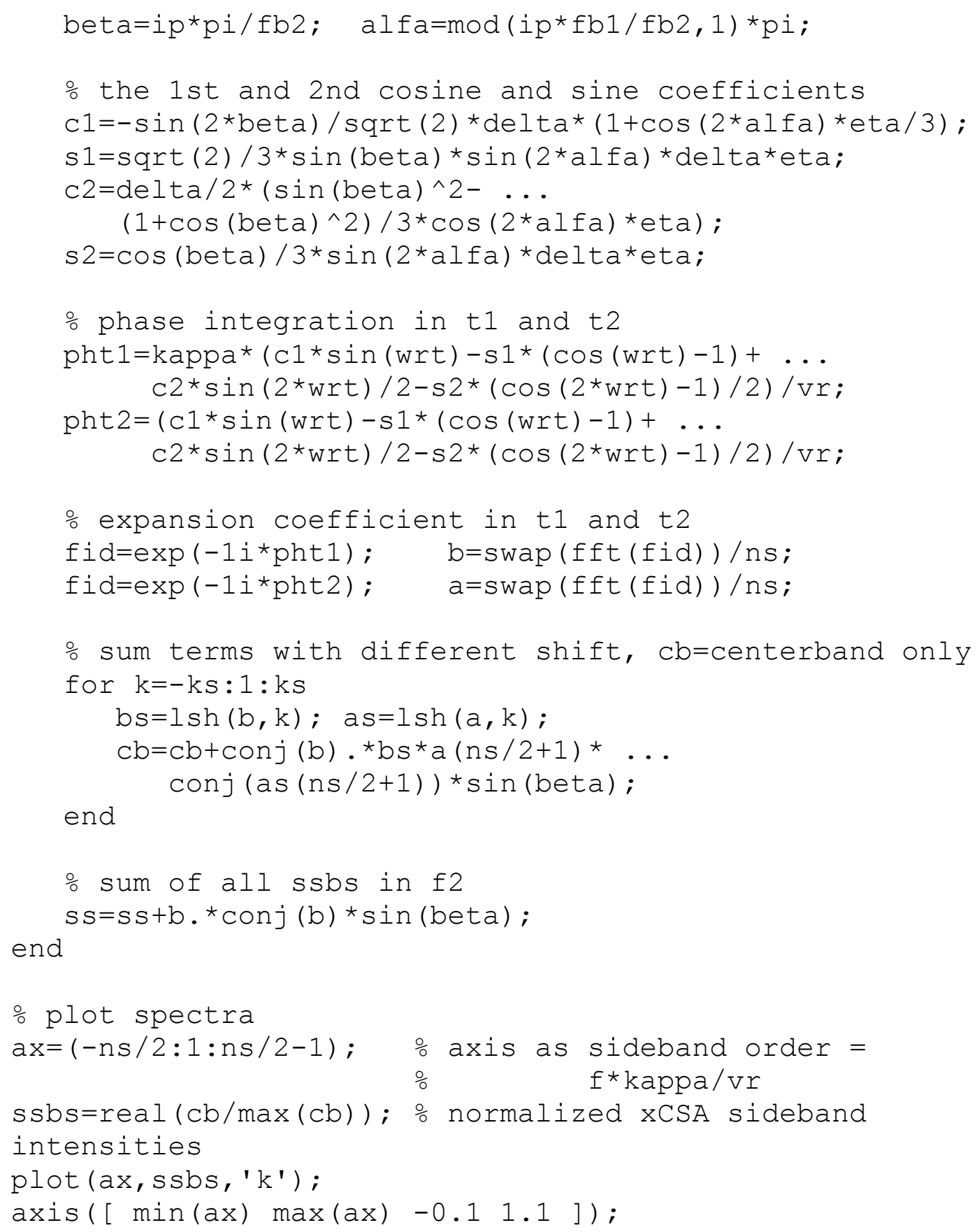



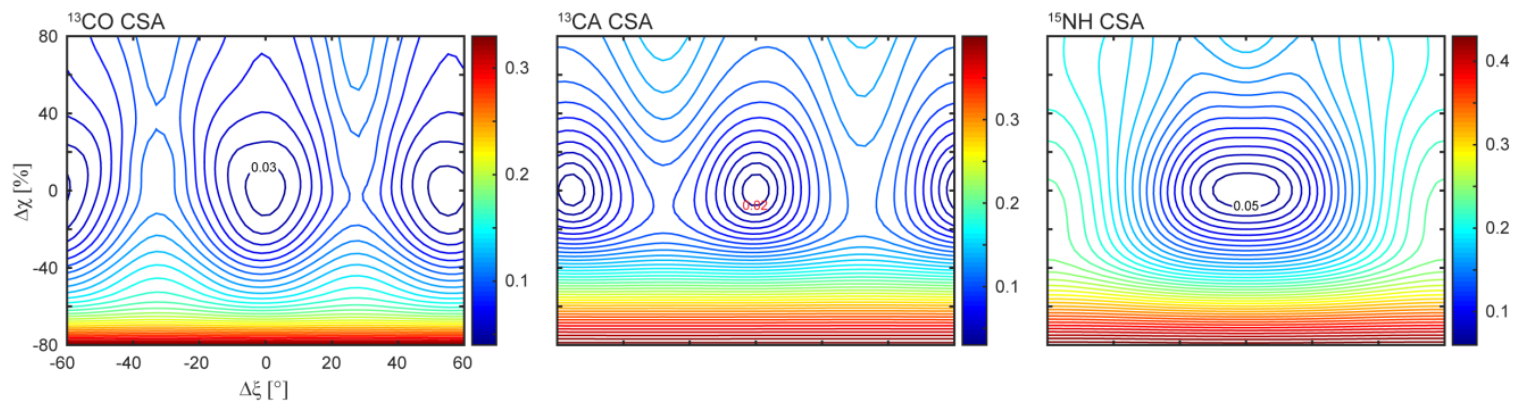

Fig. A1. Contour plots of the root-mean-square difference from the least-square fits in Fig. 4 as a function of the CSA parameters. The three traceless CSA principal components used in least-square fitting and the plots are defined as $\chi \cdot \sqrt{2 / 3}\left[\cos \left(\xi-120^{\circ}\right), \cos \left(\xi+120^{\circ}\right), \cos \xi\right]$, with $\chi=\sqrt{\left(\delta_{11}-\delta_{i s o}\right)^{2}+\left(\delta_{22}-\delta_{i s o}\right)^{2}+\left(\delta_{33}-\delta_{i s o}\right)^{2}}$ and angle $\xi$ representing the anisotropy and asymmetry of the CSA tensor. For the ${ }^{13} \mathrm{CO}$ and ${ }^{13} \mathrm{CA}$ plots, the double minima are due to a change of the order of the three principal components. 
Graphical Abstract

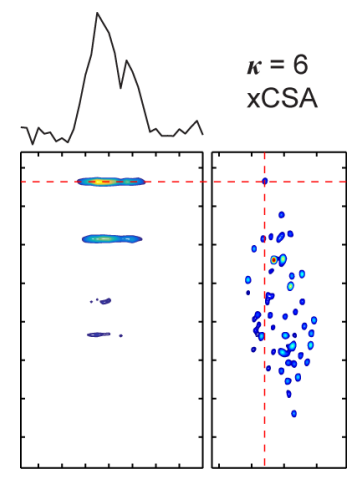

